\title{
Téoros
}

Revue de recherche en tourisme

\section{Les hébergements récréotouristiques au Québec : l'état des lieux}

\section{Véronique Barnier}

Volume 8, numéro 1, mars 1989

France-Québec

URI : https://id.erudit.org/iderudit/1080353ar

DOI : https://doi.org/10.7202/1080353ar

Aller au sommaire du numéro

Éditeur(s)

Université du Québec à Montréal

ISSN

0712-8657 (imprimé)

1923-2705 (numérique)

Découvrir la revue

Citer cet article

Barnier, V. (1989). Les hébergements récréotouristiques au Québec : l'état des lieux. Téoros, 8(1), 18-21. https://doi.org/10.7202/1080353ar d'utilisation que vous pouvez consulter en ligne.

https://apropos.erudit.org/fr/usagers/politique-dutilisation/ 


\section{Les hébergements récréo- touristiques au Québec: I'état des lieux}

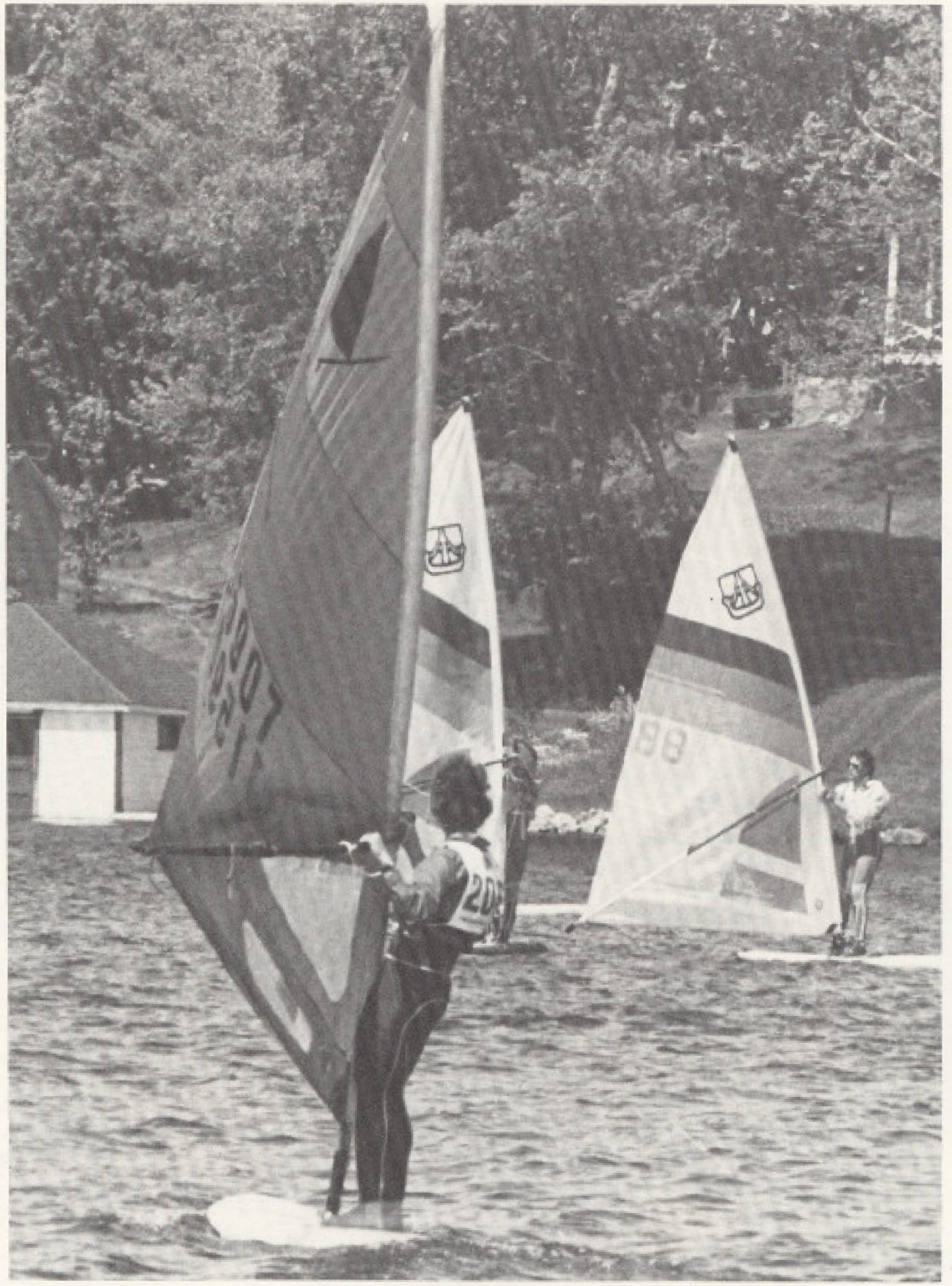

Touché à la fois par la crise économique et par la crise de l'État-Providence, le tourisme social québécois s'est retrouvé face à un contexte de privatisation et de coupures des aides gouvernementales qui ont contribué à l'ébranler sérieusement (à l'image d'ailleurs de ce qui se passe dans d'autres pays européens). Cela a continué à aggraver la situation de bon nombre d'établissements touristiques de ce secteur qui traversaient déjà une période difficile due à des problèmes internes (vétusté d'installations souvent anciennes ...).

Cependant, avant d'aller plus loin dans cette description, il est indispensable de préciser le sens de quelques notions de base en vigueur au Québec, car la situation québécoise a généré un vocabulaire bien particulier qui se différencie parfois de l'usage qu'il
- Véronicue Barnier est consultante en tourisme at a ró lisé une étude dans le cadre du Sommet québécois sur le loisir tenu en 1987. Ce texte s'en inspire. 
peut avoir ailleurs ou bien qui peut lui être totalement propre. Cette définition d'ordre sémantique est d'autant plus utile que certains termes comme "tourisme social", "loisir touristique", "tourisme associatif", sont souvent employés les uns pour les autres; en fait, ils désignent des réalités non identiques, tout en ayant des significations très proches. Cette ambiguilté dans le vocabulaire se retrouve aussi au niveau de catégories plus concrètes, utilisées dans les statistiques; ce qui entretient des confusions rendant difficiles, si ce ne n'est impossibles, toute compilation quantitative et encore plus toute comparaison chiffrée.

\section{Quelques termes en usage au Québec}

Nous allons done tenter ici de différencier le sens et l'emploi des trois concepts fourretout, mais indissociables et distincts, de tourisme social, loisir touristique et tourisme associatif, qui servent tous trois de référent aux mêmes hébergements socio-touristiques et à toute une série d'autres prestataires de services. Et, à partir de là, nous próciserons les nomenclatures utilisées au Québec concernant de manière plus concrète et spécifique les réseaux d'hébergements socio-touristiques.

Le terme même de tourisme social était peu ou pas employé jusqu'à il y a une quinzaine d'années. Il s'agit plutôt du transfert d'un concept européen. Mais ce qui, au départ, était peut-être plus une transposition qui permettait de donner une référence idéologique a l'action de certains organismes et pouvait paraître comme "plaquée" sur la situation existante, s'est révélé assez rapidement correspondre à une réalité assez proche de ce qui se passe outre-atlantique. Un certain nombre d'établissements et d'associations se réclament ainsi désormais de cette mouvance; cependant, la résonance de ce terme dans la société québécoise reste encore relativement faible et bien en-delà des deux autres.

Les mots "loisir touristique" , d'un usage beaucoup plus courant, renvoient à la scission du champ d'intervention de l'État dans le domaine touristique entre le ministère du Tourisme et le ministère du Loisir, de la Chasse et de la Pêche (MLCP) avec l'attribution du tourisme considéré comme commercial au premier et du loisir touristique au second. Il s'agit done au départ d'une définition strictement d'ordre institutionnel qui fait référence au domaine de compétence du MLCP, par différenciation avec celui du Tourisme. Alors que le terme de tourisme social renvoie plus à des références théoriques, à un projet social, source de sens et de légitimation des organismes qui s'en réclament, le loisir touristique, quant à lui, désigne des établissements, pour la majeure partie à but non lucratif, qui mettent en avant l'accessibilité aux vacances et dont la mission sociale était jusqu'à présent reconnue par l'État qui pouvait les soutenir financièrement.
Cette définition reste vaste, englobe des réalités diverses et a des limites bien floues. En effet, il peut être difficile de saisir les points communs qui existent entre tous ces organismes: le loisir touristique est plus que le loisir dit "subventionne" (II) et il n'est pas, non plus, équivalent à un type de gestion basé sur des corporations à but non lucratif? Cette difficulté à définir le loisir touristique est due au fait qu'il sous-entend l'existence artificielle d'un tourisme commercial et d"un "autre" tourisme et elle subsistera tant que l'on ne considèrera pas le tourisme comme une seule activité ayant des dimensions commerciales et sociales, les unes pouvant certes primer sur les autres.

Cela dit, très concrètement, le MLCP regroupe, pour les fins de ses programmes d'aide, les hébergements de loisir touristique en trois réseaux: les auberges de jeunesse, les bases de plein air et les camps de vacances, qui sont subdivisés selon leur clientèle majoritaire, en camps pour familles, camps pour jeunes et camps pour handicapés. Il ne faut pas se fier aux mots mêmes de camp de vacances (dérivés de l'anglais) ou de base de plein air, qui cachent des équipements en dur, parfois sophistiqués, bien éloignés de l'installation rudimentaire qu'ils laissent sous-entendre.

Le tourisme associatif, quant a lui, renvoie aux organismes regroupés en association au sein du Regroupement Loisir Québec (secteur tourisme). Les associations, dont sont membres les divers organismes gestionnaires d'hébergement socio-touristique, constituent cinq réseaux qui ne sont pas similaires aux réseaux du ministère ${ }^{(2)}$.

- le réseau des camps familiaux regroupés dans le Mouvement québécois des camps familiaux (MQCF). Les membres en sont des organismes gestionnaires de camps ou - ce qui est une de leurs particularités d'ailleurs - des groupes d'usagers;

- le réseau des camps de vacances regroupé dans l'Association des camps du Québec (ACQ):

- le réseau des bases de plein air regroupé dans le Réseau plein air;

- le réseau de l"ajisme avec la Fédération québécoise de l'ajisme, qui regroupe exclusivement les membres usagers;

- le réseau de la Fédération des Agricotours du Québec qui comprend les gîtes du passant, les fermes de vacances et d'autres formules d'hébergement en milieu rural.

Bref, tout ceci donne une petite idé de la difficulté a cerner ce qui appartient à quoi et qui est qui. Pourtant, cet exercice de recentrage, bien que fastidieux, est indispensable pour saisir le contexte problématique dans lequel évolue le tourisme social au Québec. En effet, la réalité d'un gîte rural et du tourisme rural peut sembler bien éloignée de celle d'un camp pour jeunes. Pourtant ce qui les rapproche c'est, bien sür, d'avoir une même histoire institutionnelle et le même interlocuteur gouvernemental, en l'occurrence le MLCP, mais surtout de s"être forgé une identité et un rôle social tournant autour de l'accessibilité qu'elle soit financière, territoriale ou autre.

Il ne faut pas oublier néanmoins que l'existence des hébergements socio-touristiques était paradoxalement bien antérieure à ce mouvement d'institutionnalisation et à l'apparition de ces concepts dans les années 70.

Suite a une longue tradition cléricale et religieuse qui avait mis en place, dès le début du siècle, un certain nombre d'installations, la relève fut prise par de nouveaux acteurs: groupes communautaires, État, associations... (syndicats, comités d'entreprise, collectivités locales sont par contre absents de ce tableau).

\section{Quelques données sur les hébergements socio-touristiques.}

Ces hébergements ${ }^{(3)}$ représentent environ 475 établissements, soit une capacité d'accueil proche de 34000 lits. Au niveau de la province, cela correspond à $15 \%$ de l'offre touristique globale en terme d'établissements et $8 \%$ du nombre total de lits, ce qui est loin d'être négligeable. Ces hébergements, ce sont environ 240 camps de vacances dont presque la moitié sont membres de I'ACQ, en majeure partie des camps qui recoivent des enfants ou des adolescents pendant l'été, mais également des familles ou des clientèles spécialisées (handicapés, personnes âgées...). Ce sont aussi des bases de plein air, une vingtaine dont treize sont membres de Réseau plein air, plus de 20 auberges de jeunesse et une vingtaine de camps familiaux membres du Mouvement québécois des camps familiaux. Ceci sans compter les 6 Centres de vacances familiales (CVF), la cinquantaine de fermes de vacances et les 115 gites du passant.

Il y a donc là une grande diversité de formules. Ce qui tend d'ailleurs à rendre vulnérable ce secteur économique bien que la fragmentation de l'offre en de nombreux organismes, de faible dimension la plupart du temps, soit en partie compensée par une organisation associative assez forte. Il s'agit de petites entreprises dont les budgets d'opération ne sont pas élevés puisqu'ils sont situés en moyenne autour de $200000 \$$. Il faut noter, par contre, qu'il y a de gros ccarts suivant les types d'établissement et que ces ecarts ont tendance à se creuser tragiquement. Ainsi ceux qui ont les plus petits budgets les voient diminuer (comme les auberges de jeunesse) et les budgets les plus importants se confortent de plus en plus (comme pour les bases de plein air dont les budgets atteignent $700000 \$$ en moyenne).

Pourtant, même si ce secteur économique est fragile, comme nous venons de le voir, il est une source de recettes et d'emplois non négligeable. Ainsi, uniquement en ce qui concerne 
les séjours d'été(1) dans les camps de vacances, il a généré plus de 50 millions de dollars en 1987 et produit plus de 100 millions de dollars par an de revenus consolidés. Rien que dans les hébergements subventionnés se sont vendus près d'un million de nuitées (sans compter les inscriptions journalières qui sont aussi nombreuses en règle générale que les inscriptions séjour) et la fréquentation annuelle de la totalité des camps de vacances s'élève lui à plus de 2,2 millions de jours/activité.

Voyons maintenant quelques données sur la localisation. Les hébergements touristiques (sauf les CVF et les Agricotours) sont localisés à proximité des principaux bassins de clientèle: la moitié d'entre eux sont situés dans la région administrative 06 (qui comprend Montréal, Richelieu-Rive sud et la majeure partie de Lanaudière et des Laurentides) et $13 \%$ dans la région de Québec. Cette polarisation autour de Montréal est surtout très forte pour les camps destinés aux familles, qui se retrouvent à $80 \%$ dans cette région, alors que par exemple nous y rencontrons seulement $32 \%$ des bases de plein air.

Ces zones de concentration correspondent par ailleurs à des espaces à forte vocation touristique et récréative (Laurentides et Cantons de l'Est). À plus petite echelle, on retrouve cette concentration dans des territoires plus restreints: 4 MRC (Municipalités régionales de comté) concentrent $32 \%$ des établissements et 5 municipalités $11 \%$.

Cette polarisation ne doit pas faire sousestimer le rôle et le poids de ces équipements en région car, parallèlement à cette concentration, il y a dispersion dans toute la province: 81 \% des MRC ont au moins un hébergement de vacances sur leur territoire!

\section{Les clientèles}

Il existe deux types de clientèle principale: les jeunes et les familles. L'intérêt pour la clientèle familiale est plus récent et a été marqué par l'apparition de nouveaux produits (parfois sous impulsion gouvernementale comme les CVF). Cette part du marché est actuellement en train de s'accroître et semble devancer la clientèle plus traditionnelle des jeunes. Un certain nombre d'études ont été réalisées pour saisir le profil socioéconomique des usagers des hébergements socio-économiques et ainsi mesurer en quelque sorte leur degré social. Mais les résultats ne sont pas identiques suivant les réseaux.

Pour les camps familiaux membres du $\mathrm{MQCF}^{(5)}$, l'objectif social semble atteint comme l'indiquent les résultats suivants: $58 \%$ des usagers ont des revenus bruts familiaux de moins de $20000 \$, 52 \%$ ne sont pas actifs sur le marché du travail et $62 \%$ n'ont pas atteint le niveau collégial au plan de la scolarisation (à noter que $76 \%$ sont des femmes et $37 \%$ sont parents de familles monoparentales).
Quant à la clientèle des camps de vacances pour jeunes ${ }^{(6)}$, elle reste fortement correlée depuis plusieurs années aux différents types d'établissements: les jeunes des camps subventionnés proviennent de familles qui ont des revenus moins élevés que ceux des camps sans but lucratif non subventionnés, qui euxmêmes ont des revenus moins élevés que la clientèle des camps à but lucratif. Dans les bases de plein air ${ }^{(7)}$, la clientèle est de plus en plus scolarisée et la "structure des professions a tendance à se mouvoir vers le haut de la pyramide, tout en se démarquant de l'ensemble de la population québécoise". II y a recentrage vers les classes moyennes pour les CVF également (donc une clientèle beaucoup plus sollicitée par le secteur dit commercial).

Il apparait donc que la fréquentation de certains types d'hébergement se déplace vers des couches sociales plus éduquées, ayant des revenus plus élevés. Mais il $n^{*} e n$ reste pas moins que les camps familiaux et les camps de vacances, pour une grande partie, desservent une clientèle plus défavorisée. Un détail qui a son importance: il n'y a pas, comme en France, de marché des collectivités ( $s i$ ce ne sont les écoles); on ne peut dire qu'il existe un marché du tourisme social avec des caractéristiques très précises, autres que les traditionnelles variables socio-economiques. Le marché des hébergements sociotouristiques correspond plus à une fraction de l'ensemble du marché touristique (ce qui lui donne peut-être plus d'atouts lorsqu'il s'agit de se confronter aux lois du marché).

Une attention particulière doit ćtre portée cependant à la clientèle des jeunes placés par les centres des services sociaux (CSS). Mais, même là, le prix du sejour de l'enfant est celui en vigueur dans le camp; le seul critère déterminant le choix d'un camp (autre que la qualité du service et la sécurité dans le camp) est qu'il n'y ait pas plus de $30 \%$ de clientèle provenant d'un CSS $^{(8)}$

Enfin, il faut noter une diversification de plus en plus grande de la clientèle dans les hébergements de vacances. Ainsi, dans les bases de plein air, il y a de plus en plus de familles, de moins en moins d'adultes (individuels) et de scolaires; dans les camps pour familles par contre, la part des familles diminue passant de $78 \%$ en 83 à $62 \%$ en 87 . Dans les camps pour jeunes, la part des adultes, elle, augmente: inexistante en 83 , elle représente $13 \%$ de la clientèle en 87 ; et même dans les camps pour handicapes, la part des handicapés diminue de $79 \%$ en 83 à $50 \%$ en 87. Quant aux auberges de jeunesse, elles sont fréquentées par des personnes de plus en plus âgées ( $57 \%$ de plus de 25 ans en 85 ) et ont une notoriété de plus en plus faible auprès des jeunes de 15-20 ans.

La spécialisation de chacun s'érode au fil des années. Si le phénomène est général, les causes n'en sont pas moins différentes certainement d'un réseau à l'autre: symptôme de crise, d'inadaptation du produit, fruit d'une stratégie commerciale, survie pour la recherche de nouvelle clientèle plus lucrative? Autant de questions qui peuvent être soulevées et que nous ne ferons qu'esquisser.

Voici done, rapidement brossé, l'état des lieux socio-touristiques au Québec. Mais un certain nombre de problèmes risquent actuellement de modifier profondément la structure de ce secteur économique et laissent présager un avenir tout ce qu'il y a de plus incertain.

\section{Une aide gouvernementale qui tend à disparaître}

L'État a reconnu et reconnait toujours la mission sociale - permettre à un plus grand nombre de partir en vacances - mise en avant par toute cette sphère d'établissements touristiques (pour la majeure partie à but non lucratif). Ce soutien se traduit par une politique d'assistance financière du MLCP qui s'élevait à près de $3250000 \$$ en 1987-1988.

Mais ces programmes (tant au niveau de l'aide au fonctionnement que de l'aide à l'investissement, l'aide à la personne n'existant pas) connaissent depuis quelques années de sérieuses coupures ( $34 \%$ entre 1984 et 1988 soit de 5 millions de \$ a 3 1/4 millions) qui remettent en question le fragile équilibre financier de bon nombre d'organismes. Cette baisse est très forte pour les auberges de jeunesse $(-70 \%)$ qui ne reçoivent plus du tout d'aide au fonctionnement depuis l'année budgétaire 1987-1988. L'enveloppe destinée aux bases, camps familiaux et camps pour jeunes a diminué de près du tiers sur ces quatre années-là, pendant que seules les subventions aux camps pour handicapés se maintenaient.

Les bases, elles, ont signé une entente avec le ministère pour négocier les conditions d'un retrait des aides au fonctionnement: étalement sur 5 ans de ce retrait, compensé par une aide de $50000 \$ / a n$ dans des budgets spécifiques de promotion dans le but d'aider les bases à assurer leur capacité d'autofinancement.

D'autres types de modification ont eu lieu pendant ces mêmes dernières années comme l'assouplissement des critères d'admissibilité (abolition du plafond à la tarification) ou l'abandon de l'aide pour la réalisation des plans directeurs, l'abandon de l'aide pour l'achat d'équipement de loisir. Les CVF occupent une place à part dans ce tableau. En effet, ils n'ont jamais recu d'aide au fonctionnement mais par contre ont bénéficié dans les premières années de leur existence d'un budget global de $1020000 \$$ réservé à des actions d'immobilisation, d'organisation administrative ou de promotion. Ce programme expérimental a servi à créer et organiser ces nouveaux équipements (qui ne reçoivent plus aucune aide depuis) dans un cadre défini par le ministère.

Les effets, pour des etablissements qui dépendaient des subventions, peuvent être 
catastrophiques car les conditions de survic pouvaient être liées à cette aide. Que penser par exemple de $\mathrm{l}^{\dagger}$ avenir des camps pour les familles, pour qui la part des revenus de subvention en 1984-1985 représentait $56 \%$ de leurs revenus totaux d'opération? Que penser aussi de celui des camps pour handicapés où cette part s'élève à $46 \%$; à $37 \%$ pour les camps de jeunes et a $26 \%$ pour les bases?

Bien sûr ce ministère n'a pas l'exclusivité des coupures et les organismes reçoivent de moins en moins d'assistance financière quelle qu'elle soit. Les autres types de subventions peuvent provenir d'organismes publics (OPDQ, féderal, dans leurs programmes d'aide au développement régional ou à la création d'emplois), de fondations privées, religieuses ou non, ou d'organismes communautaires comme Centraide Montréal. Ce dernier a d'ailleurs formulé au début 1988 une nouvelle politique d'intervention ${ }^{(9)}$. Cette réorganisation de l'aide financière de Centraide Montréal se fait autour d'un resserrement des modalités d'attribution afin de: "rendre les ressources financées accessibles aux plus démunis de notre société"t. Pour cela les camps subventionnés devront se doter d'une échelle de tarification proportionnelle et accueillir $20 \%$ de démunis.

Ces multiples retraits de subvention désćquilibrent financièrement bon nombre d'organismes et risquent de modifier leur vocation première: l'objectif d'accessibilitế que certains s'efforçaient de maintenir est remis en cause directement, l'autofinancement passant par une hausse obligée de la tarification.

\section{Le loisir touristique au pied du mur}

Les comportements de vacances de la clientèle québécoise sont sujets à des changements profonds. Comme dans beaucoup de pays occidentaux, les nouvelles tendances du marché vont vers un fractionnement des vacances, une réduction de la durée des séjours, une multiplication des départs lors des longs week-ends, un développement des forfaits tout compris, etc... Face à ces nouveaux besoins, les hébergements socio-touristiques doivent s'adapter et être prêts à offrir des produits parfois différents dans un Québec qui subit des transformations socio-démographiques profondes (accroissement des familles mono-parentales, vieillissement de la population, poids grandissant des groupes ethniques, taux de fécondité très bas...).

Ceci est d'autant plus crucial que le marché des vacances est devenu très compétitif (cf la guerre des prix que se livrent les tour operators sur le marché du sud) alors que la destination même du Québec ${ }^{(10)}$ comme lieu de vacances pour les Québécois est en perte de vitesse. Désormais, l'autofinancement passe par le positionnement sur le marché des vacances, le ciblage de nouvelles clientèles et la redéfinition des produits. Cette démarche peut être d'autant plus difficile à acquérir pour des organismes qui avaient une distribution centrée sur la vente directe mais une politique de mise en marché peu agressive et une stratégie de marketing inexistante.

Cependant, ces problèmes ne leur sont pas spécifiques du tout; que l'on se réfere aux diagnostics alarmants qui sont portés actuellement sur le tourisme québécois ${ }^{(11)}$; gros écarts saisonniers des activités, non qualification de la main-d'oeuvre, promotion faible et non articulée, etc... Étonnamment, les hébergements socio-touristiques ont parfois de meilleurs résultats que l'hôtellerie traditionnelle (en termes de taux d'occupation). Tous les hébergements n'accueillent pas obligatoirement, loin s'en faut, une clientele défavorisée et tous ne remplissent pas des objectifs sociaux. Mais il est certain que beaucoup d'entre eux (qui sont en règle générale subventionnés) offrent la possibilité de partir en vacances à des Québécois qui ne le feraient pas sans cela. Et ce sont eux qui sont par définition les premiers visés par les coupures ministérielles alors que l'objectif d'accessibilité sociale répond à un besoin qui n'a pas pour autant disparu de la société québécoise (ainsi le maintien du taux de non départ en vacances).

Tout se passe comme si les organismes étaient confrontés au double choix suivant: soit chercher à résoudre les problèmes d'autofinancement par une hausse des tarifs, soit maintenir à tout prix l'objectif social. Or ces défis, tous ne pourront certainement pas les relever. La cause essentielle ne réside pas dans une absence d'expertise ou dans une quelconque incapacité inhérente mais plus prosaiquement dans. l'inadaptation d'équipements vieillis. La chose est grave: la plupart des installations sont anciennes, considérées souvent comme vétustes: elles ne correspondent plus aux normes de la nouvelle Loi sur les établissements touristiques (mise en place par le ministère du Tourisme) auxquelles elles vont désormais être soumises. Là est le problème central. Les travaux nécessaires à cette mise aux normes sont évalués (12), rien que pour les camps de vacances, à 90 millions de $\$$ (ce qui ne comprend ni les coûts relatifs à la simple modernisation des équipements ni à l'adaptation aux nouveaux besoins).

C"est à partir d'une telle analyse de la situation que le groupe de travail sur le loisir touristique a formulé un certain nombre de recommandations auprès du MLCP dans le but d'instaurer une véritable politique d'accessibilité aux vacances. En ce qui concerne les réseaux d'hébergement, elle reste centrée prioritairement sur les objectifs suivants: conserver le patrimoine immobilier que constituent tous les hébergements sociotouristiques par un programme d'aide aux biens immobiliers et aux équipements, maintenir la vocation sociale de certains établissements à but non lucratif par un programme d'aide au fonctionnement et reconnaitre le principe de l'aide à la personne. On peut toutefois se demander si ces mesures seront suffisantes pour enrayer l'état de crise des hébergements les plus atteints. f

\section{Notes}

(1) II est intéressant de noter que seuhement le quart des établissements de loisir touristique sont subventionnés (bien qu'ils rêprégentent près de la moite $\{47 \%$ ) de la capacité d'accucil en nombre de lits! ce qui tend ả montrer que la majeure partie des établissements do loisir touristique fonctionne sans aucune aicle du MLCP. A titre de comparaison, le sectuur du ski alpin a reçu des subwentions de 35 millions $\$$ ces quatre dernièras années.

(2) Les camps familiaux ont un sens précis pour le RLO, alors que pour le ministère, ils revoient à tout camp ayant majoritairement des familles comme clientitye. Ainsi les camps pour famille, tels que définis par le MLCP, peuvent être membres de l'ACO etiou du MQCF.

(3) La majeure partie des données provient de l'étude réalisée pour le groupe de travail.

141 Une strategie de mise en marché pour les camps de vacances. Juin 1988. MLCP.

(5) Jean Stafford et Pierre Bellerose, Profil socioúconomique et mesure de la satisfaction des usagers dans les établissements membres du MOCF. Montréal, Université du Québec à Montréal (UDAM), Avril 88.

(6) id. (4)

(7) Jean Stafford, Etude et analyse socio. économique de la clientêle des bases membres du Réseau plein air, Montréal, UQAM, Mai B8.

(8) Le programme camps de vacances du ministere des Affaires Sociales a representé une enveloppe de 1731000 \$ en 1987 pour tout le Québec, répartie dans les 14 CSS; elle est aflectét aux camps de vacances sous forme d'aide directe at la personne, calculke suivant un per diem.

(9) If finance actuellement 47 organismes de vacances dont 30 sur une base tenouvelable $(21$ camps de vacances +8 groupes de vacances + MOCF pour 1153300 s on 1950 - 1987 (aide au fonctionnement) ce qui correspond à une contribution de $8,80 \$$ par personne/jour.

[10] Le nombre de voyages/personnes au Ouébec (touristes d'agrément québicoisl a diminué de $24 \%$ entre 1980 et 1996. P, Cluzeau, Indicateurs sur les marchés et sur les secteurs touristiques de 1980 à 1986, ministère du Tourisme.

(11) Samson-Búlair, Planification stratégique du tourisme québécois, document provisoire, 13 septembre 1988. Québec, ministére du Tourisme.

(12) Groupe DBSF, Etude şur les plans de développement des camps de vacances, Québuc, MLCP. Juin 1988. 